\title{
BMJ Open Interventional radiology versus operative management for splenic injuries: a study protocol for a systematic review and meta-analysis
}

Masahiro Kashiura, ${ }^{\oplus 1}$ Noritaka Yada, ${ }^{2}$ Kazuma Yamakawa ${ }^{\circledR}$

To cite: Kashiura M, Yada N, Yamakawa K. Interventional radiology versus operative management for splenic injuries: a study protocol for a systematic review and meta-analysis. BMJ Open 2019;9:e028172. doi:10.1136/ bmjopen-2018-028172

- Prepublication history and additional material for this paper are available online. To view these files, please visit the journal online (http://dx.doi org/10.1136/bmjopen-2018028172).

Received 25 November 2018 Revised 11 April 2019 Accepted 23 July 2019
Check for updates

(C) Author(s) (or their employer(s)) 2019. Re-use permitted under CC BY-NC. No commercial re-use. See rights and permissions. Published by BMJ.

${ }^{1}$ Department of Emergency and Critical Care Medicine, Saitama Medical Center, Jichi Medical University, Saitama, Japan ${ }^{2}$ Department of General Medicine, Nara Medical University, Kashihara, Japan ${ }^{3}$ Division of Trauma and Surgical Critical Care, Osaka General Medical Center, Osaka, Japan

Correspondence to Dr Masahiro Kashiura kashiura@me.com

\section{ABSTRACT}

Introduction Over the past decades, the treatment for blunt splenic injuries has shifted from operative to nonoperative management. Interventional radiology such as splenic arterial embolisation generally increases the success rate of non-operative management. However, the type of intervention, such as the first definitive treatment for haemostasis (interventional radiology or surgery) in blunt splenic injuries is unclear. Therefore, we aim to clarify whether interventional radiology improves mortality in patients with blunt splenic trauma compared with operative management by conducting a systematic review and meta-analysis.

Methods and analysis We will search the following electronic bibliographic databases to retrieve relevant articles for the literature review: Medline, Embase and the Cochrane Central Register of Controlled Trials. We will include controlled trials and observational studies published until September 2018. We will screen search results, assess the study population, extract data and assess the risk of bias. Two review authors will extract data independently, and discrepancies will be identified and resolved through a discussion with a third author where necessary. Data from eligible studies will be pooled using a random-effects meta-analysis. Statistical heterogeneity will be assessed by using the MantelHaenszel $\chi^{2}$ test and the $\mathrm{I}^{2}$ statistic, and any observed heterogeneity will be quantified using the $\mathrm{I}^{2}$ statistic. We will conduct sensitivity analyses according to several factors relevant for the heterogeneity.

Ethics and dissemination Our study does not require ethical approval as it is based on the findings of previously published articles. This systematic review will provide guidance on selecting a method for haemostasis of splenic injuries and may also identify knowledge gaps that could direct further research in the field. Results will be disseminated through publication in a peer-reviewed journal and presentations at relevant conferences. PROSPERO registration number CRD42018108304.

\section{INTRODUCTION}

Blunt abdominal trauma is regularly encountered in the emergency department, ${ }^{1}$ and the spleen is among the most commonly injured intra-abdominal organs. ${ }^{23}$ The primary goal for blunt abdominal trauma is the diagnosis
Strengths and limitation of this study

- We will conduct a systematic review and meta-analysis of the effects of interventional radiology and operative management on mortality in patients with blunt splenic injuries using appropriate methodologies and quality assessment tools.

- We will also perform subgroup analyses according to the presence of shock, another site of trauma except for the spleen and classification of splenic injuries to compare and evaluate the efficacies of interventional radiology versus operative management.

- The results of this systematic review and meta-analysis will be highly dependent on the quality of the included primary research studies; many observational studies might be included.

and prompt management of potentially life-threatening haemorrhage. Therefore, emergent splenectomy remains a lifesaving measure for many patients.

Haemodynamically stable blunt splenic injuries are normally managed non-operatively to avoid the morbidity of unnecessary surgery and the risk of overwhelming postsplenectomy sepsis. ${ }^{4}$ The current Eastern Association for the Surgery of Trauma practice management guidelines recommend initial non-operative management (NOM) for all haemodynamically healthy patients. ${ }^{5}$ However, in some cases, a higher risk of failure of NOM is predicted, and identification and management of these patients is challenging. ${ }^{5}$ In NOM of blunt splenic injuries, interventional radiology such as splenic arterial embolisation (SAE) is used as an adjunct to observation to increase the success rate of NOM. ${ }^{6} \mathrm{NOM}$ with SAE is more favourable than observational management alone in severe splenic injuries. ${ }^{78}$ However, the challenge is in identifying the cases of severe splenic injuries in which interventional radiology or operative management is more 
appropriate than observational management alone. ${ }^{9}$ The management approach may vary from institution to institution depending on the resources, haemodynamic status of the patient, grade of splenic injury and presence of other injuries and medical comorbidities. ${ }^{510} 11$ The effect of interventional radiology on mortality of blunt splenic injuries compared with surgical management remains unclear.

Therefore, we aim to clarify if, compared with surgery, interventional radiology improves mortality in patients with blunt splenic trauma, by conducting a systematic review and meta-analysis.

\section{METHODS AND ANALYSIS}

\section{Protocol registration}

This study protocol has been registered in PROSPERO (http://www.crd.york.ac.uk/PROSPERO/). ${ }^{12}$ The protocol follows the Preferred Reporting Items for Systematic Reviews and Meta-Analyses Protocols (PRISMA-P) statements, ${ }^{13} 14$ and the systematic review and meta-analysis will be reported according to the PRISMA statement (see online supplementary file 1). ${ }^{1516}$

\section{Database searches}

We will search the following major electronic bibliographic databases to retrieve relevant articles for the literature review: Medline (Ovid), Embase (Proquest) and The Cochrane Central Register of Controlled Trials. Search terms will include 'splenic injury', 'spleen rupture', 'interventional radiology', 'endovascular procedures' and 'therapeutic embolisation'. We will consult a librarian for the database search. We will assess the references of relevant articles to determine if additional studies can be found. The detail of the search strategy is available in the online supplementary file 2 .

\section{Types of studies}

We will include controlled trials (including randomised controlled trials and other controlled trials) and observational studies (including prospective and retrospective cohort studies, case-control studies and before-after studies) that reported data on our primary or secondary outcomes published until September 2018. Studies will be excluded if they did not clearly report the population, treatment or outcomes of interest. Animal studies will be excluded. Grey literature such as conference proceedings and abstracts will be included. If two or more studies were published using the same or overlapping cohorts, the most recent or larger cohort will be included. No language restrictions will be applied. We will handle non-English language publications using appropriate translation services.

\section{Study population}

We will include adult patients (ie, those aged $\geq 16$ years) admitted to the hospital with blunt splenic injuries requiring invasive intervention for haemostasis, such as interventional radiology or surgery. We will not restrict our review by country and will include all types of splenic injuries. Patients with penetrating abdominal trauma, patients without invasive intervention, and paediatric patients will be excluded.

\section{Intervention and control}

Intervention types of interest will be SAE as the initial definitive treatment for haemostasis, including any embolisation technique (distal embolisation, proximal splenic artery embolisation or a combination) and the material used for embolisation (coils, gelatin sponge, or n-butyl-2-cyanoacrylate). Meanwhile, comparators will be any operative management such as splenorrhaphy, partial splenectomy and splenectomy as the initial definitive treatment for haemostasis including both laparoscopic approach and open abdominal exploration.

\section{Outcomes}

The primary outcome will be in-hospital mortality due to all causes. Secondary outcomes will be 30 day mortality, further intervention, complications such as abdominal compartment syndrome and infection, length of intensive care unit stay and length of hospital stay.

\section{Data extraction}

Citations will be stored, and duplicates will be removed using EndNote software (Thomson Reuters, Toronto, Ontario, Canada). We will use Rayyan software for the systematic review process. ${ }^{17}$ Titles and abstracts of studies retrieved using the search strategy will be screened independently by two review authors (MK and NY) to identify studies that potentially meet the inclusion criteria. The full text of these potentially eligible studies will be retrieved and assessed independently by two review authors (MK and NY). Any disagreement about the eligibility of studies will be resolved through a consult with a third reviewer (KY). The flow chart of the study, which is based on the PRISMA statement, ${ }^{16}$ is shown in figure 1 .

Data from the included studies will be extracted for assessment of study quality and data synthesis using a standardised prepiloted form. The extracted information will include the following: study setting; study population and participant baseline characteristics; details of the intervention and control conditions; study methodology; outcomes and times of measurement and information for the assessment of risk of bias. Two review authors (MK and $\mathrm{NY}$ ) will extract data independently, and discrepancies will be resolved through a discussion with the third author $(\mathrm{KY})$. Authors will not be contacted to request the provision of any missing data.

\section{Assessment of risk of bias in individual studies}

Independent reviewers (MK and NY) will assess the risk of bias in individual studies as methodological quality of articles, and disagreements will be resolved by a discussion with a third reviewer $(\mathrm{KY})$. We will apply uniform criteria for evaluating the risk of bias associated with individual randomised control trials based on the Cochrane risk of 


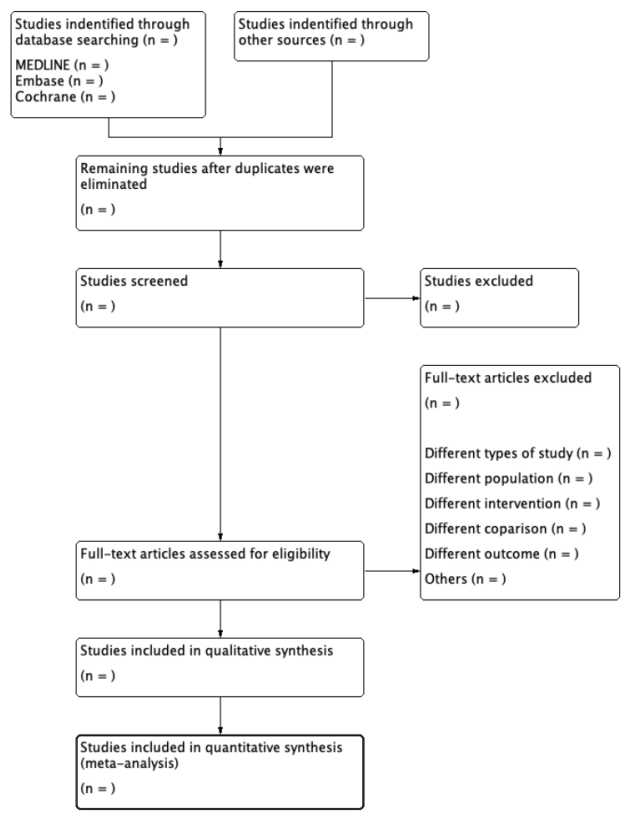

Figure 1 Flowchart of the study selection process.

bias tool. ${ }^{18}$ Each study will be assessed for (1) random sequence generation, (2) allocation concealment, (3) blinding of participants and personnel, (4) blinding of related outcomes assessment, (5) incomplete outcome data, (6) selective reporting and (7) other bias. We will also apply the Risk of Bias Assessment Tool for Nonrandomized Studies (RoBANS) to assess the risk of bias of observational studies, which is compatible with the Cochrane risk of bias tool. ${ }^{19}$ In particular, there is a potential risk of time-dependent bias, including immortal time bias, in eligible studies. We will evaluate how appropriate statistical methods, such as marginal structural models, have been used in each eligible study, to manage time-dependent bias.

\section{Summarising data}

We will perform a meta-analysis when data are available in one or more trials according to the 'Cochrane Handbook for Systematic Reviews of Interventions'. For dichotomous variables (eg, mortality, need for further intervention and complication of abdominal compartment syndrome and infection), values for the risk ratio (RR) or the OR will be expressed as a point estimate with $95 \%$ CIs. Continuous outcomes, such as length of intensive care unit stay and length of hospital stay, will be expressed as their mean difference with $95 \%$ CIs and $p$ value. If quantitative synthesis is not appropriate for a particular outcome, we will provide a qualitative summary.

\section{Data synthesis}

We will provide estimates of the findings from the included studies according to a random-effect model. A random-effect model incorporates statistical heterogeneity and provides a more conservative estimate of the pooled effect size than a fixed-effect model. We will not perform multiple imputation for missing data. We will perform data synthesis and analysis of randomised trials and observational studies separately.

All statistical analyses including the risk of bias within studies and across studies will be performed with Review Manager V.5.3. (RevMan; The Cochrane Collaboration 2014, The Nordic Cochrane Centre, Copenhagen, Denmark) and STATA software V.14.0 (Stata Corporation, College Station, Texas, USA). The statistical significance will be set at a $p$ value of $<0.05$.

\section{Assessment of heterogeneity}

Statistical heterogeneity will be assessed via the MantelHaenszel $\chi^{2}$ test and the $\mathrm{I}^{2}$ statistic as the proportion of total variability explained by heterogeneity. Substantial heterogeneity is predefined as a $p$ value of $<0.10$ with the Mantel-Haenszel $\chi^{2}$ test or an $\mathrm{I}^{2}$ value of $>50 \% .^{20}$ Possible sources for heterogeneity will be investigated via meta-regression analysis. The log $\mathrm{RR}$ of death in the interventional radiology group will be plotted against the control mortality rate for each study. A linear meta-regression weighted to reflect the variance of the individual studies will be used to model the data. The presence of clinical heterogeneity will be considered in the decision to conduct a quantitative synthesis of data or to perform sensitivity analyses, as below.

\section{Sensitivity and subgroup analysis}

We will examine the robustness of the meta-analyses by conducting sensitivity analyses according to different components of the Cochrane risk of bias tool or RoBANS, as appropriate. If the necessary data are available, subgroup analyses will be done according to the country, sample size, publication year (published in the last 10 years vs published 10 years ago or longer), presence of shock at admission, other site of trauma except for the spleen and American Association for the Surgery of Trauma classification of splenic injuries. ${ }^{21}$

\section{Assessment of reporting bias}

To assess publication bias, we will create funnel plots for mortality in which the log RRs will be plotted against their SEs, and we will test the symmetry of the funnel plots using the Begg's rank correlation test and Egger's linear regression test. ${ }^{22}$

\section{Rating the certainty of evidence using the GRADE approach}

Two authors (MK and NY) will independently assess the strength of evidence using the Grading of Recommendations Assessment, Development and Evaluation (GRADE) approach. ${ }^{23}$ The quality of evidence will be assessed for each outcome and categorised as high, moderate, low or very low according to the GRADE approach.

\section{Trial sequential analysis}

Cumulative meta-analyses have the potential risk of type I errors due to repeated significance test. ${ }^{24}$ Therefore, we will apply trial-sequential analysis. Trial-sequential analysis combines information size estimation for meta-analysis with an adjusted threshold for statistical 
significance in the cumulative meta-analysis. Information size will be calculated as diversity-adjusted information size, suggested by the relative risk reduction of the intervention in the included trials. ${ }^{25}{ }^{26}$ In principle, strong evidence is likely to be established if the trial sequential monitoring boundary is crossed before reaching the required information size. If this boundary is not crossed, it is highly likely that trials will need to be continued. ${ }^{27}$

\section{Patient and public involvement}

This study will not involve any patient or the public.

\section{DISCUSSION}

Determining the appropriate type of intervention, that is, surgery versus interventional radiology, for haemostasis in blunt splenic injuries remains a challenge for emergency physicians, trauma surgeons and interventional radiologists. Moreover, the influence of interventional radiology on the mortality of blunt splenic injuries compared with operative management remains unclear. Therefore, we will conduct a systematic review and meta-analysis of the effects of interventional radiology compared with surgery on mortality in patients with blunt splenic injuries.

Several observational studies have demonstrated that NOM is more successful with the adjunctive use of SAE. ${ }^{28-34}$ NOM is performed in over $50 \%$ of haemodynamically stable patients without increasing mortality even in grade 4 or 5 splenic injuries. ${ }^{2835}$ The success rates for SAE vary depending on institutional policies, embolisation technique, arterial accessibility, interventional radiologist skill and the type of embolisation material. ${ }^{36} 37$

Several systematic reviews and meta-analyses of patients with blunt splenic injuries have been reported. ${ }^{7-9}$ Two studies compared NOM with and without SAE. ${ }^{7}$ The results showed that SAE is associated with significantly higher splenic salvage rates in grades 4 and 5 splenic injuries. ${ }^{78}$ SAE significantly reduced the failure of NOM in patients with grades 4 and 5 splenic injuries but had minimal effect in those with grades $1-3$ injuries. ${ }^{8}$ Meanwhile, one study compared NOM (including with and without splenic arterial management) and operative management. ${ }^{9} \mathrm{NOM}$ was associated with decreased mortality in minor splenic injuries. ${ }^{9}$ However, integrating the results for severe splenic injuries was difficult because of the substantial heterogeneity and potentially inappropriate comparison groups. ${ }^{9}$

The results of the above studies indicate that NOM with SAE is more favourable than observational management alone in severe splenic injuries. ${ }^{78}$ However, the challenge is in determining the appropriate intervention for haemostasis in severe splenic injuries (interventional radiology vs operative management), not observational management alone. To the best of our knowledge, no systematic review and meta-analysis has directly compared the effect of interventional radiology including SAE with that of operative management. Our systematic review and meta-analysis will be the first study focused on interventional radiology versus operative management. This systematic review and meta-analysis will provide current evidence for researchers in this field and useful information for treatment of patients with blunt splenic injuries.

\section{ETHICS AND DISSEMINATION}

Our findings will be presented at relevant scientific conferences and disseminated through publication in a peer-reviewed journal.

Acknowledgements We would like to thank Takaaki Suzuki of Nara Medical University Library (Nara, Japan) for his assistance to conduct the database search. We would also like to thank Editage (www.editage.jp) for English language editing.

Collaborators Takaaki Suzuki.

Contributors MK, NY and KY contributed to the conception of the study. The manuscript protocol was drafted by MK and was revised by NY and KY. The search strategy was developed by all of the authors and will be performed by MK. MK and NY will also independently screen the potential studies, extract data from the included studies, assess the risk of bias and complete the data synthesis. KY will arbitrate in cases of disagreement and ensure the absence of errors. All authors approved the publication of this protocol.

Funding This work was supported by the ZENKYOREN (National Mutual Insurance Federation of Agricultural Cooperatives).

Competing interests None declared.

Patient consent for publication Not required.

Ethics approval Approval from an ethics committee is not required because this systematic review will use publicly available data without directly involving human participants.

Provenance and peer review Not commissioned; externally peer reviewed.

Data availability statement There are no data in this work.

Open access This is an open access article distributed in accordance with the Creative Commons Attribution Non Commercial (CC BY-NC 4.0) license, which permits others to distribute, remix, adapt, build upon this work non-commercially, and license their derivative works on different terms, provided the original work is properly cited, appropriate credit is given, any changes made indicated, and the use is non-commercial. See: http://creativecommons.org/licenses/by-nc/4.0/.

\section{REFERENCES}

1. Nishijima DK, Simel DL, Wisner DH, et al. Does this adult patient have a blunt intra-abdominal injury? JAMA 2012;307:1517-27.

2. Isenhour JL, Marx J. Advances in abdominal trauma. Emerg Med Clin North Am 2007;25:713-33.

3. Davis JJ, Cohn I, Nance FC. Diagnosis and management of blunt abdominal trauma. Ann Surg 1976;183:672-8.

4. Cullingford GL, Watkins DN, Watts $A D$, et al. Severe late postsplenectomy infection. Br J Surg 1991;78:716-21.

5. Stassen NA, Bhullar I, Cheng JD, et al. Selective nonoperative management of blunt splenic injury: an eastern association for the surgery of trauma practice management guideline. J Trauma Acute Care Surg 2012;73(5 Suppl 4):S294-300.

6. Bhangu A, Nepogodiev D, Lal N, et al. Meta-Analysis of predictive factors and outcomes for failure of non-operative management of blunt splenic trauma. Injury 2012;43:1337-46.

7. Requarth JA, D'Agostino RB, Miller PR. Nonoperative management of adult blunt splenic injury with and without splenic artery embolotherapy: a meta-analysis. J Trauma 2011;71:898-903.

8. Crichton $\mathrm{JCl}$, Naidoo K, Yet B, et al. The role of splenic angioembolization as an adjunct to nonoperative management of blunt splenic injuries: a systematic review and meta-analysis. J Trauma Acute Care Surg 2017;83:934-43.

9. Cirocchi R, Boselli C, Corsi A, et al. Is non-operative management safe and effective for all splenic blunt trauma? A systematic review. Crit Care 2013;17.

10. Hildebrand DR, Ben-Sassi A, Ross NP, et al. Modern management of splenic trauma. BMJ 2014;348:g1864. 
11. Peitzman AB, Heil B, Rivera L, et al. Blunt splenic injury in adults: multi-institutional study of the eastern association for the surgery of trauma. J Trauma 2000;49:177-89.

12. Moher D, Booth $A$, Stewart L. How to reduce unnecessary duplication: use Prospero. BJOG: Int J Obstet Gy 2014;121:784-6.

13. Shamseer L, Moher D, Clarke M, et al. Preferred reporting items for systematic review and meta-analysis protocols (PRISMA-P) 2015: elaboration and explanation. BMJ 2015;349:97647-7.

14. Moher D, Shamseer L, Clarke M, et al. Preferred reporting items for systematic review and meta-analysis protocols (PRISMA-P) 2015 statement. Syst Rev 2015;4.

15. Liberati A, Altman DG, Tetzlaff J, et al. The PRISMA statement for reporting systematic reviews and meta-analyses of studies that evaluate health care interventions: explanation and elaboration. PLoS Med 2009;6:e1000100.

16. Moher D, Liberati A, Tetzlaff J, et al. Preferred reporting items for systematic reviews and meta-analyses: the PRISMA statement. PLoS Med 2009;6:e1000097.

17. Ouzzani M, Hammady H, Fedorowicz Z, et al. Rayyan-a web and mobile APP for systematic reviews. Syst Rev 2016;5:210.

18. Higgins JPT, Altman DG, Gøtzsche PC, et al. The Cochrane collaboration's tool for assessing risk of bias in randomised trials. BMJ 2011;343:d5928.

19. Kim SY, Park JE, Lee YJ, et al. Testing a tool for assessing the risk of bias for nonrandomized studies showed moderate reliability and promising validity. J Clin Epidemiol 2013;66:408-14.

20. Huedo-Medina TB, Sánchez-Meca J, Marín-Martínez F, et al. Assessing heterogeneity in meta-analysis: $Q$ statistic or $\mathrm{I}^{2}$ index? Psychol Methods 2006;11:193-206.

21. Moore EE, Cogbill TH, Jurkovich GJ, et al. Organ injury scaling: spleen and liver (1994 revision). J Trauma 1995;38:323-4.

22. Sterne JAC, Sutton AJ, loannidis JPA, et al. Recommendations for examining and interpreting funnel plot asymmetry in meta-analyses of randomised controlled trials. BMJ 2011;343:d4002.

23. Guyatt $\mathrm{GH}$, Oxman $A D$, Vist GE, et al. Grade: an emerging consensus on rating quality of evidence and strength of recommendations. BMJ 2008;336:924-6.

24. Imberger G, Thorlund K, Gluud C, et al. False-Positive findings in Cochrane meta-analyses with and without application of trial sequential analysis: an empirical review. BMJ Open 2016;6:e011890.
25. Brok J, Thorlund K, Gluud C, et al. Trial sequential analysis reveals insufficient information size and potentially false positive results in many meta-analyses. J Clin Epidemiol 2008;61:763-9.

26. Afshari A, Wetterslev J, Brok J, et al. Antithrombin III in critically ill patients: systematic review with meta-analysis and trial sequential analysis. BMJ 2007;335:1248-51.

27. Wetterslev J, Thorlund K, Brok J, et al. Trial sequential analysis may establish when firm evidence is reached in cumulative meta-analysis. $J$ Clin Epidemiol 2008;61:64-75.

28. Haan JM, Biffl W, Knudson MM, et al. Splenic embolization revisited: a multicenter review. J Trauma 2004;56:542-7.

29. Banerjee A, Duane TM, Wilson SP, et al. Trauma center variation in splenic artery embolization and spleen salvage: a multicenter analysis. J Trauma Acute Care Surg 2013;75:69-75

30. Liu PP, Lee WC, Cheng YF, et al. Use of splenic artery embolization as an adjunct to nonsurgical management of blunt splenic injury. $J$ Trauma 2004;56:768-73.

31. Rajani RR, Claridge JA, Yowler CJ, et al. Improved outcome of adult blunt splenic injury: a cohort analysis. Surgery 2006;140:625-32.

32. Sabe AA, Claridge JA, Rosenblum DI, et al. The effects of splenic artery embolization on nonoperative management of blunt splenic injury: a 16-year experience. J Trauma 2009;67:565-72.

33. Miller PR, Chang MC, Hoth JJ, et al. Prospective trial of angiography and embolization for all grade III to V blunt splenic injuries: nonoperative management success rate is significantly improved. $J$ Am Coll Surg 2014;218:644-8.

34. Capecci LM, Jeremitsky E, Smith RS, et al. Trauma centers with higher rates of angiography have a lesser incidence of splenectomy in the management of blunt splenic injury. Surgery 2015;158:1020-6.

35. Olthof DC, van der Vlies $\mathrm{CH}$, Goslings JC. Evidence-Based management and controversies in blunt splenic trauma. Curr Trauma Rep 2017;3:32-7.

36. Raikhlin A, Baerlocher MO, Asch MR, et al. Imaging and transcatheter arterial embolization for traumatic splenic injuries: review of the literature. Can J Surg 2008;51:464-72.

37. Bhullar IS, Frykberg ER, Tepas JJ, et al. At first blush: absence of computed tomography contrast extravasation in grade IV or V adult blunt splenic trauma should not preclude angioembolization. $J$ Trauma Acute Care Surg 2013;74:105-11. 